\title{
Early Adversity and the Prospective Prediction of Depressive and Anxiety Disorders in Adolescents
}

\author{
Nicole K. Phillips, ${ }^{1}$ Constance L. Hammen, ${ }^{1,4}$ Patricia A. Brennan, ${ }^{2}$ \\ Jake M. Najman, ${ }^{3}$ and William Bor ${ }^{3}$
}

Received November 3, 2003; revision received April 6, 2004; accepted July 12, 2004

\begin{abstract}
The current study was a prospective exploration of the specificity of early childhood adversities as predictors of anxiety and depressive disorders in adolescents. Participants were 816 adolescents (414 males, 402 females) with diagnostic information collected at age 15; information on early adversities had been collected from the mothers during pregnancy, at birth, age 6 months, and age 5 years for a related study. Adolescents with "pure" anxiety disorders were compared with adolescents with "pure" depressive disorders (major depressive disorder, dysthymia), and these groups were compared to never-ill controls. Analyses controlled for gender and maternal depression and anxiety disorders. Results indicated that adolescents with anxiety disorders were more likely than depressed youth to have been exposed to various early stressors, such as maternal prenatal stress, multiple maternal partner changes, and more total adversities, whereas few early childhood variables predicted depressive disorders. Even when current family stressors at age 15 were controlled, early adversity variables again significantly predicted anxiety disorders. Results suggest that anxiety disorders may be more strongly related to early stress exposure, while depressive disorders may be related to more proximal stressors or to early stressors not assessed in the current study.
\end{abstract}

KEY WORDS: depression; anxiety; early adversity; longitudinal.

Kessler and Magee (1993) investigated the power of retrospectively reported childhood adversities occurring through age 16 to predict depression in an epidemiological sample. Eight adversities were studied, and many were found to be associated with incidence and prevalence of major depressive disorder. Parental drinking, parental mental illness, violence in the family, parents' marital problems, death of mother or father, and absence of a close adult relationship in childhood were significantly associated with onset of depression by age 20 . These results are consistent with psychological models of depression that commonly emphasize the influence of negative childhood experiences in the family affecting the qual-

\footnotetext{
${ }^{1}$ Department of Psychology, University of California, Los Angeles, California.

${ }^{2}$ Emory University, Atlanta, Georgia.

${ }^{3}$ University of Queensland, Brisbane, Australia.

${ }^{4}$ Address all correspondence to Constance L. Hammen, Department of Psychology, University of California, Los Angeles, California 90095; e-mail: hammen@psych.ucla.edu.
}

ity of the relationship between child and parents, and exposing the child to stressful experiences that overwhelm his or her capacities to cope effectively, creating vulnerability to develop depressive episodes in adolescence or adulthood. For instance, poor maternal care, physical or sexual abuse, parental marital discord, exposure to family violence, parental loss, and parental mental illness or substance abuse have all been linked to childhood and adult depression (reviews in Burbach \& Borduin, 1986; Goodman, 2002; Hammen, 1991; Kaslow, Deering, \& Racusin, 1994).

However, childhood adversities such as poor parenting, parental marital discord, parental mental illness, and childhood abuse are predictive of a broad range of psychological disorders (e.g., Coie et al., 1993; Johnson, Cohen, Kasen, Smailes, \& Brook, 2001; Repetti, Taylor, $\&$ Seeman, 2002). Indeed, most studies of the association between childhood environmental conditions and later depression failed to test whether the association is specific to depression compared to another form of disorder. Kessler, 
Davis, and Kendler (1997) addressed the specificity issue in data from the National Comorbidity Survey. The study obtained participant reports of 26 childhood adversitiesfive interpersonal loss events, eight interpersonal traumas, and four forms of parental psychopathology, separately for mothers and fathers, and miscellaneous adversities including natural disasters. The adversities were retrospectively reported to have occurred by age 16 . The investigators found that most of the adversities were significantly related to all the broad classes of participant disorder (depression, anxiety, substance, and antisocial disorders). Moreover, Kessler et al. (1997) noted that results were attenuated when comorbid effects of other disorders were controlled. Thus, they emphasized that future studies need to control for the effects of comorbidity and to examine whether there are specific early environmental predictors of particular forms of disorder.

The purpose of the present study is to begin to address the specificity issue with respect to early predictors of anxiety disorders and depressive disorders, two internalizing disorders that are insufficiently distinguished in the early adversity literature. Although less has been written about the precursors of anxiety disorders than depressive disorders, several studies have shown that retrospectively reported adverse events such as sexual or physical abuse, and family disruptions (variously defined as parental emotional abuse or neglect, marital conflict, parent substance abuse or mental illness) were related to anxiety disorders in community (Brown \& Harris, 1993; Laraia, Stuart, Frye, Lydiard, \& Ballenger, 1994; Levitan, Rector, Sheldon, \& Goering, 2003; Spence, Najman, Bor, O'Callaghan, \& Williams, 2002; Tweed, Schoenbach, George, \& Blazer, 1989; reviewed by Fredman, HirshfeldBecker, Smoller, \& Rosenbaum, 2003; Rapee, 1997) and clinical samples (Young, Abelson, Curtis, \& Nesse, 1997).

In addition to the relative paucity of research on specificity of anxiety vs. depression associated with childhood adversity, most childhood adversity research is also limited by retrospectively recalled information about childhood circumstances, and by a broad age of exposure (often, any time before age 16). Moreover, there is relatively little guidance about conceptual bases for prediction of depression vs. anxiety outcomes. Studies of adults by Finlay-Jones and Brown (1981) and Beck, Brown, Steer, Eidelson, and Riskind (1987) have generally supported the hypothesized association between depression and perceived loss, and between anxiety and perceived threat. However, such constructs have not been used to characterize childhood adversity experiences, or to link such experiences specifically with depression or anxiety outcomes.
The present study addresses the question of whether certain specific childhood adversities may be predictive of anxiety or depressive disorders, employing data from a longitudinal study that included contemporaneous measures of adversity variables and later diagnostic evaluations to age 15 in a large community sample of youth. The original study from which data for the current work are taken was not designed specifically for the current questions, but information was available about certain stressful conditions affecting the child's family life and environmental circumstances up to age 5 . We focused on five domains from the data available in early childhood, making an effort to select variables, if available, that corresponded to previously reported measures of adversity (e.g., Kessler \& Magee, 1993) and those noted in anxiety or depression research as mentioned previously: marital relations (mothers' relationships with partner), economic hardship, parental deviance, maternal stressful life events, and early childhood health problems. The latter topic has been explored in several studies of children's anxiety disorders or internalizing symptoms, with some, although not all, studies finding an association between early-onset chronic medical problems and anxiety (Allen, Lewinsohn, \& Seeley, 1998; Bandelow et al., 2002; Cohen, Velez, Brook, \& Smith, 1989; Gortmaker, Walker, Weitzman, \& Sobol, 1990; Kellerman, Zeltzer, Ellenberg, Dash, \& Rigler, 1980). The adversities to be included in the present analyses represent a variety of potential loss or threat occurrences for young children, but the actual perceptions of the children were not available for study.

The present study is, thus, an exploratory study that attempts further refinement of the specificity issue by examining the association of early childhood adversities (to age 5) and early onset (prior to age 15) of "pure" depressive (major depressive episode or dysthymic disorder) and "pure" anxiety disorders (omitting from the sample those who had comorbid anxiety/depressive disorders), compared to a nonill control group. The present report draws from a community sample of 15-year-old youth and their mothers who were selected for a study originally designed to address outcomes of adolescent children of depressed and never-depressed women (the Queensland High Risk Project). Over 800 families were selected from a large birth cohort study [the Mater-University Study of Pregnancy (MUSP)] originally devised to investigate children's physical, cognitive, and psychological health as a function of pregnancy and obstetric conditions, birth weight, and psychosocial conditions, and to predict age 5 health, development and behavior. MUSP data were thus available to evaluate early adversity, and also served as the basis for selection of the later Queensland High Risk Project. Therefore, data collected in two separate 
investigations with different purposes were combined to yield a unique longitudinal prospective study including variables collected in early childhood and at age 15 .

Exploratory hypotheses, guided by past research, include the prediction that negative marital/relationship, maternal stress, and poverty factors predict both depressive and anxiety disorders, although whether the association is stronger for one group or the other is unclear. Child physical illness variables are predicted to be more strongly associated with anxiety than depressive disorders. All associations are evaluated controlling for history of maternal depression or anxiety disorders. To rule out the possibility that associations between early adverse conditions and later symptomatology are attributable to the possibly chronic, continuing nature of the early stressors under investigation, further analyses were conducted controlling for the level of chronic stress at age 15.

\section{METHODS}

\section{Participants}

The current sample consisted of a birth cohort of 816 adolescents, 414 males (50.7\%) and 402 females (49.3\%), born at the Mater Misericordiae Mothers' Hospital in Queensland, Australia, between 1981 and 1984. The participants were originally part of the MUSP, which followed a larger cohort of 7,775 children, 4,038 males $(51.9 \%)$ and 3,737 females $(48.1 \%$ ), from birth through age 5 (Keeping et al., 1989). In the MUSP, families were assessed four times, at approximately 18 weeks gestation, 3-5 days after the birth of the child, at 6-months old, and at 5-years old. Overall, 6846 of the mothers identified themselves as Caucasian (88.1\%), 277 as Asian (3.6\%), and 292 as Maori or Australian Aborigine (3.8\%). The median family income was in the range of lower middle socioeconomic status. In the original sample, $71.5 \%$ of the mothers reported completing grade 10 or less.

In the original birth cohort sample, mothers selfreported depression symptoms on the Delusion States Symptom Inventory (DSSI; Bedford, Foulds, \& Sheffield, 1976) at the four time points indicated above. On the basis of mothers' scores representing diverse (or no) experiences of depressive symptoms, a smaller subset of 816 mothers and their children was selected for followup at youth age 15 for the Queensland High Risk Project (P. Brennan \& C. Hammen, investigators). The current analyses are based on the 15 years of data collected on the sample of 816 families. Approximately two-thirds of the mothers had some degree of depression, as verified by lifetime history of mood disorders based on diagnostic interviews assessed when the child was 15 as noted below. Specifically, 85 mothers $(10.4 \%)$ met criteria for a current diagnosis of major depressive disorder or dysthymia, and $354(43.4 \%)$ reported a past history of depression.

The mean age of the adolescents at follow-up was 14.9 -years old $(S D=.37)$. According to the mothers' reports of ethnicity, $729(89.3 \%)$ of the adolescents were Caucasian, 8 (1.0\%) were Asian, $6(.7 \%)$ were Maori or Aborigine, and 51 (6.2\%) were of mixed descent. Overall, the families in this sample represented a lower middle class to lower class SES.

Compared to the children not selected for follow-up, the current sample did not differ on the basis of gender $\left[\chi^{2}\right.$ $(1, N=7775)=.53, p=.47, n s]$, family income $\left[\chi^{2}(6\right.$, $N=7149)=1.61, p=.95, n s]$, or mother's education level $\left[\chi^{2}(7, N=7622)=6.42, p=.49, n s\right]$. The current sample had fewer ethnic minority members than the original sample $\left[\chi^{2}(4, N=7438)=8.87, p=.07\right]$.

The sample of 816 adolescents was initially classified into three groups based on diagnosis: a depressive disorder group (13.5\% of the total), an anxiety disorder group (12.3\%), and a control group (74.2\%). Twenty-eight adolescents with comorbid anxiety-depression disorders (25\% of the depressed youth had anxiety disorders and $28 \%$ of those with anxiety disorders also had depressive disorders) were excluded from the analyses. To control for potential effects of other comorbidities, an additional 97 adolescents ( $12.9 \%$ of the total) were also eliminated from the current analyses for meeting diagnostic criteria for ADHD, conduct disorder, oppositional-defiant disorder, eating disorder, or a substance use disorder (65 from the control group, 21 from the depressive disorder group, and 11 from the anxiety disorder group).

The resulting depressive disorder-only group consisted of 61 adolescents with a lifetime history of either dysthymia or major depressive disorder. Of those adolescents, $12(19.7 \%)$ had a current diagnosis of a depressive disorder, and $60(98.4 \%)$ had past diagnoses of depression. The anxiety disorder-only group consisted of 61 adolescents with a lifetime history of panic disorder, obsessivecompulsive disorder, generalized anxiety disorder, posttraumatic stress disorder, social phobia, or specific phobia. Among adolescents in that group, 35 (57.4\%) currently met criteria for one or more anxiety disorder diagnoses and $48(78.7 \%)$ have past anxiety diagnoses. The control group consisted of 569 adolescents who had no disorders. In analyses to be reported, sample sizes may vary due to missing data on individual variables from the early childhood data. 


\section{Procedure}

In the initial phase of the MUSP, women at their first prenatal clinic visit at the Mater Misericordiae Public Hospital were invited to participate in a study on pregnancy. After giving consent to participate, the women were asked to complete a 117-item self-report questionnaire on health problems, psychosocial factors, daily activities, and their attitudes toward their pregnancy. Approximately 35 days after their child's birth, the women were asked to complete a second 103-item self-report questionnaire that assessed changes that occurred during pregnancy. When the child reached the age of 6 months, the mothers were mailed a 103-item questionnaire assessing changes since the previous wave. The researchers used telephone reminders as well as home visits to improve response rates. Obstetric data were also collected from hospital records and medical charts. When the child reached the age of 5, the mothers were contacted and asked to complete an additional set of self-report questionnaires assessing health and psychosocial variables for both themselves and their child. Further details about the procedures used during the initial study are reported by Keeping et al. (1989).

When the child reached the age of 15 , families selected for inclusion in the current study were contacted and asked to participate in a follow-up to the original MUSP. Interviewers traveled to the families' homes at a time that was convenient for both the adolescent and the mother to conduct a 3.5-hour interview. After obtaining consent from the participants, trained clinicians, blind to maternal status on the DSSI, administered diagnostic and other semistructured interviews to both the adolescents and their parents. Participants then completed a series of self-report questionnaires on a laptop computer. For their participation, parents received AU\$20 and adolescents received AU\$10.

\section{Measures}

\section{Schedule for Affective Disorders and Schizophrenia in School-Aged Children}

The Schedule for Affective Disorders and Schizophrenia in School-Aged Children (K-SADS-E; Orvaschel, 1995) is a semistructured interview for diagnosing current presence or past history of Axis I psychiatric disorders for children and adolescents. Trained clinicians administered the interview to both the adolescents and their mothers. Best estimate diagnoses were assigned by the clinical interview team, typically giving most weight to information, if discrepant across informants, from the most credible source (Leckman, Sholomskas, Thompson, Belanger, \&
Weissman, 1982). In the present study, adolescents diagnosed with anxiety disorders reported a history of generalized anxiety disorder, obsessive-compulsive disorder, panic disorder, posttraumatic stress disorder, social phobia, and/or specific phobia. Adolescents with depressive disorders reported a history of either major depressive disorder or dysthymia.

Seventy-five of the KSADS-E interview tapes were randomly selected for reliability ratings by a second clinician blind to the original diagnoses. Overall, the weighted kappa for depressive disorders was .82 for current disorders and .73 for past disorders. The weighted kappa for anxiety disorders was .76 for current disorders and .79 for past disorders.

\section{Structured Clinical Interview for DSM-IV}

The Structured Clinical Interview for DSM-IV (SCID; First, Spitzer, Gibbon, \& Williams, 1995) is a semistructured interview, administered by trained clinicians, which is used to diagnose current and past Axis I psychiatric disorders. The SCID was administered to the mothers in the sample to obtain a history of maternal psychopathology. For the current study, mothers were categorized as depressed $(N=125)$ if they met full criteria for either major depressive disorder or dysthymia during the child's first 5 years of life. Mothers were categorized as anxious $(N=33)$ if they met full criteria for panic disorder, generalized anxiety disorder, obsessive-compulsive disorder, or posttraumatic stress disorder during the child's first 5 years.

To determine interrater reliabilities in the current sample, 52 of the SCID interview tapes were randomly selected for rating by a second clinician blind to the original diagnoses. Overall, the weighted kappa for past maternal depressive disorders was .84, and the weighted kappa for past maternal anxiety disorders was .85. Previous maternal substance abuse had a weighted kappa of .88. All other past maternal disorders, including eating disorders, had a kappa of .97 .

\section{Childhood Adversity Measures}

Data on adverse conditions before the age of 5 were taken from the various maternal self-report questionnaires administered during the first four waves of data collection in MUSP (prenatal, postnatal, 6 months, 5 years). Adversity was operationalized for the present study as mothers' reports of negative circumstances across five domains: mother's relationship with partner, economic hardship, early childhood health problems, parental deviance, and maternal stressful life events. 
Adversities that involved mothers' relationships with their partners were operationalized as partner separations, partner changes, and overall quality of the relationship. At the fourth wave of data collection, the mothers reported on two questions, whether she had been separated, and whether she had made a partner change during the previous 5 years. Each of those variables was then coded as "yes" or "no." The quality of the mother's relationship with her current partner was obtained at each of the four waves of data collection from the mother's selfreport on the eight satisfaction items of the Dyadic Adjustment Scale (DAS; Spanier, 1976). Alphas at each wave of data collection for the DAS were $.86, .85, .88$, and .97 , respectively.

Economic hardship was operationalized by aggregating mothers' self-report of total family income over three of the first four waves of data collection. At the prenatal, 6 month, and 5 year waves, mothers reported average family income on a 7-point scale. Responses were averaged across the time points, and those in approximately the lowest $10 \%$ of the distribution of average incomes were identified as experiencing consistent poverty.

Early childhood health problems over the past 5 years were assessed from maternal reports in two areas at the age-5 assessment. Mothers were asked "has your child had any of these conditions or symptoms continuing longer than three months" (items included asthma, seizures, anemia, heart defect, cancer, and other serious conditions), and they rated each on a 3-point scale (1-not present for 3 months, 2-present and limited child's activities somewhat, 3-present and limited activities a lot). Presence of impairing chronic illnesses was defined as a maternal report indicating that the child's daily activities were impacted either "some" or "a lot" by one or more illnesses. Mothers also indicated the number of times the child was hospitalized before age 5; history of multiple hospitalizations was defined as having two or more hospitalizations.

Parental deviance was operationalized as mothers' reports of their own and their partner's troubles with the police, based on the questionnaire completed at child age 5-years-old. Maternal deviance and partner deviance were each defined as endorsing "been in trouble with the police" one or more times during the previous 5 years.

Maternal prenatal and postnatal stressors were assessed with a shortened life events checklist consisting of 9 items at the prenatal assessment and 8 items at the postnatal assessment. The checklist included items covering significant disagreements, health problems, financial problems, and work stress. At each of the two time points, the stress variable consisted of the number of items endorsed from the list.

\section{Current Family Chronic Stress}

Chronic stress (ongoing conditions over at least the past 6 months) was assessed through semistructured interview with the mothers, covering typical functioning in key roles (e.g., Hammen et al., 1987). The instrument yields information that is both a measure of chronic stress in these areas, and a measure of current functioning in the past 6 months. The interview includes probes for several areas reported in this study: quality of intimate (marital) relationships, relationship with the youth, and family finances. Each domain is rated by the interviewer on a 5-point scale with behaviorally specific anchor points indicating severity of ongoing stressful conditions, ranging from exceptionally good conditions (1) to extreme adversity (5). Interrater reliabilities based on independent judges' ratings ( $N=77-100$, depending on category) included intraclass correlations of .88 for current marital or intimate relationship, .82 for relationship with child, and .90 for finances. Convergent and construct validation have been obtained in various samples (e.g., Hammen et al., 1987; Rao, Hammen, \& Daley, 1999).

\section{RESULTS}

In order to evaluate potential confounding relationships between demographic factors and diagnosis, the three groups were compared on gender, ethnicity, and family income. There were significant differences in the distribution of males and females across the diagnostic and control groups, $\chi^{2}(2, N=691)=14.23, p<.01$. There were higher proportions of females in both the depressive disorder group $(N=44,72.1 \%)$ and the anxiety disorder group $(N=37,60.7 \%)$ than in the control group $(N=277,48.7 \%)$. Comparisons between diagnosis groups reported below will therefore control for gender. No significant differences among the groups were found for ethnicity, $\chi^{2}(10, N=675)=3.63, p=.96$, $n s$. A trend toward a significant difference in family income at age 15 across the diagnostic groups was noted in the sample, $F(2,666)=2.76, p=.06$, with both the depressive disorder and anxiety disorder groups reporting marginally lower mean family incomes compared to the control group. However, the anxiety and depressive disorder groups did not differ significantly, $t(116)<1$.

Because the study involving the current sample had been selected to oversample mothers with a history of depression, both maternal history of a diagnosable depressive disorder and maternal history of a diagnosable anxiety disorder present during the child's first 5 years of life were controlled in analyses in order to rule out the effects of maternal conditions on reports of early adversity and 
on youth outcomes. Maternal depression during the first 5 years was significantly related to both adolescent depressive disorders, $\chi^{2}(1, N=712)=8.82, p<.01$, and adolescent anxiety disorders, $\chi^{2}(1, N=702)=4.02, p=$ .05 . Maternal anxiety during the first 5 years was not significantly related to adolescent depressive disorders $\chi^{2}(1, N=715)=0.06, p=.81, n s$; however, it was marginally related to adolescent anxiety disorders $\chi^{2}(1$, $N=705)=2.71, p=.10$.

Correlations among the selected adversity variables are presented in Table I. Significant intercorrelations among adversities, especially among variables capturing the quality of the mother's marital relationship, were found, but these correlations were generally small.

Table II presents the frequencies of the adversity variables across the diagnostic groups. These overall comparisons indicate especially high rates of adversities for the anxiety group, and overall significant differences between groups on many of the variables. Specific comparisons between depressed and anxious groups are reported below.

\section{Differences Between Anxiety Disorder and Depressive Disorder Groups}

Table III presents logistic regression analyses predicting group status from early adversities, controlling for gender, maternal depression in the child's first 5 years, and maternal anxiety in the child's first 5 years. Results are summarized in Table III, comparing depressive vs. anxiety disorder outcomes, as well as comparisons of each diagnostic group with the control group.

The first column of odds ratios compares anxiety disorders vs. depressive disorder outcome. Results indicate that mother-reported partner changes significantly differentiated between anxiety and depressive disorders, $\chi^{2}(1, N=121)=8.79, p<.01$, such that youth with a history of maternal partner change were seven times more likely to develop an anxiety disorder than a depressive disorder (OR $=7.46,95 \% \mathrm{CI}=1.56-35.72)$. Similarly, maternal prenatal relationship satisfaction significantly differentiated between the two groups, $\chi^{2}(1, N=117)=$ $4.43, p=.04$, such that mothers of adolescents with anxiety disorders reported less satisfaction than mothers of adolescents with depressive disorders, $(\mathrm{OR}=0.95,95 \%$ $\mathrm{CI}=0.90-1.00$ ).

Maternal partner deviance also significantly differentiated between the groups, $\chi^{2}(1, N=121)=4.44, p=$ .04 , such that adolescents whose mothers reported higher levels of partner criminality were two to three times more likely to develop an anxiety disorder than a depressive disorder $(\mathrm{OR}=2.46,95 \% \mathrm{CI}=1.05-5.77)$. Overall, the total number of early adversities summed across the adversity domains significantly differentiated between the anxiety and depressive disorder groups, $\chi^{2}(1, N=119)=8.33$, $p<.01$, such that adolescents with anxiety disorders experienced more adversities than adolescents with depressive disorders $(\mathrm{OR}=2.00,95 \% \mathrm{CI}=1.20-3.34)$.

The second and third columns of odds ratios represent logistic regression comparisons of each diagnostic group with the control group, controlling for gender and both maternal depression and anxiety disorders during the first 5 years. Results indicated that only postnatal maternal stress significantly predicted depressive disorders compared to controls, $\chi^{2}(1, N=604)=4.12, p=.04$, such that children of mothers who experienced higher numbers of stressors immediately after the child's birth were more likely to develop a depressive disorder by age $15(\mathrm{OR}=$ $1.21,95 \% \mathrm{CI}=1.01-1.44)$. No other early childhood adversities significantly predicted outcomes of depressive disorders, although there was a trend toward higher postnatal stress associated with greater likelihood of depressive disorders.

In contrast, several variables were associated with youth anxiety disorders, compared to controls. Turbulence in the mothers' relationships with their partners showed significant associations with the children's development of anxiety disorders. Changing partners by the mother before the child was 5 -years old was significantly associated with anxiety disorders, $\chi^{2}(1, N=615)=10.07$, $p<.01$, and children were more than three times as likely to develop an anxiety disorder if their mother had changed partners $(\mathrm{OR}=3.67,95 \% \mathrm{CI}=1.74-7.72)$. Maternal marital satisfaction both prenatally and across the first 6 months were also significant predictors of later anxiety disorders. Low marital satisfaction during the prenatal period significantly predicted anxiety disorders by adolescence, $\chi^{2}(1, N=599)=9.21, p<.01, \mathrm{OR}=0.94$, $95 \% \mathrm{CI}=0.90-0.98$. Anxiety disorders by adolescence were also significantly predicted by lower maternal marital satisfaction when the child was 6-months old, $\chi^{2}(1, N=$ $587)=6.77, p<.01, \mathrm{OR}=0.94,95 \% \mathrm{CI}=0.90-0.98$. Adolescents with anxiety disorders were also nearly twice as likely to have had their mother's partner involved with the police, $\chi^{2}(1, N=604)=3.90, p=.05$, OR $=1.81$, $95 \% \mathrm{CI}=1.02-3.22$. Although not significant, maternal report of separation from partner before age 5 was a marginal predictor of an anxiety disorder diagnosis by adolescence, $\chi^{2}(1, N=618)=3.59, p=.06, \mathrm{OR}=$ $1.87,95 \% \mathrm{CI}=1.00-3.51$.

Additionally, maternal stressful life events appeared to be significant predictors of later anxiety disorders among their children. Higher maternal report of prenatal stressors significantly predicted anxiety disorders by adolescence, $\chi^{2}(1, N=610)=5.72, p=.02$, such that 


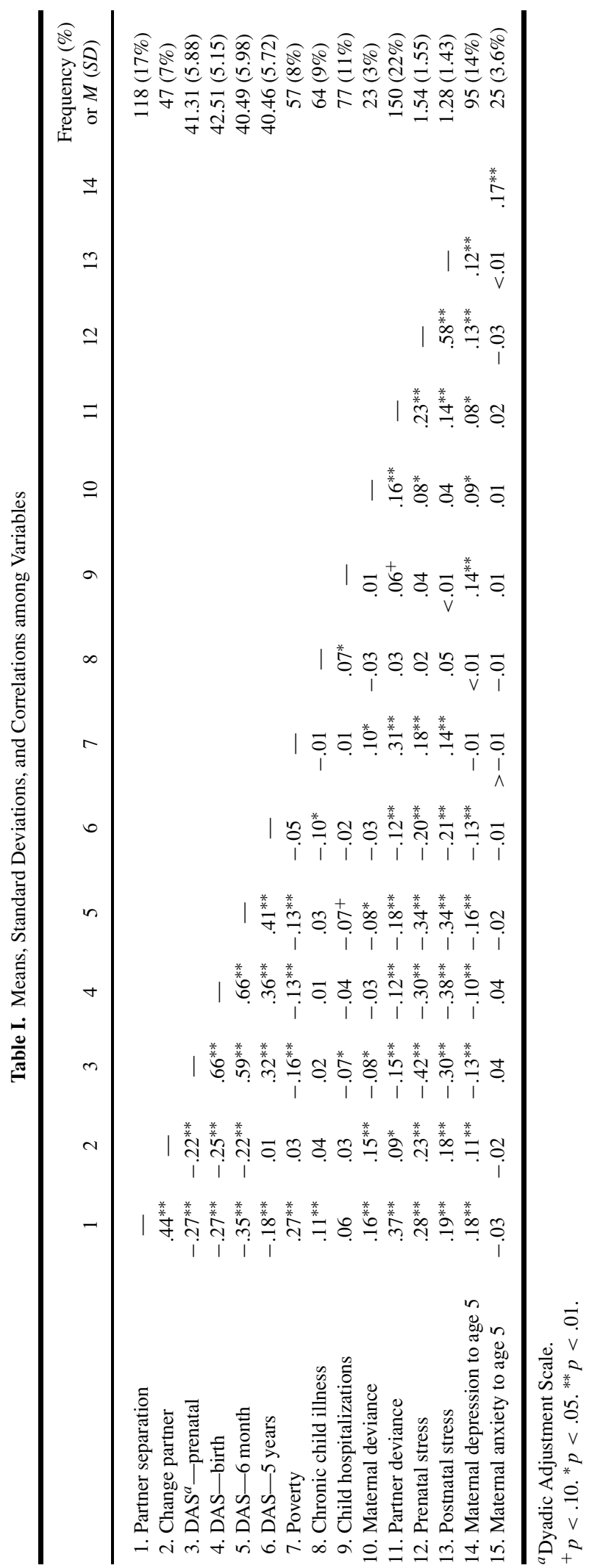


Table II. Frequencies of Adversity Variables within the Sample by Diagnostic Group

\begin{tabular}{|c|c|c|c|c|}
\hline \multirow[b]{2}{*}{ Variable } & \multicolumn{3}{|c|}{ Diagnostic groups } & \multirow[b]{2}{*}{ Test statistic } \\
\hline & Depression $(N=61)$ & Anxiety $(N=61)$ & Control $(N=569)$ & \\
\hline Partner separation & $11(18.0 \%)$ & $17(27.9 \%)$ & $90(15.8 \%)$ & $\chi^{2}(2, N=681)=6.21^{* *}$ \\
\hline Change partner & $2(3.2 \%)$ & $12(19.7 \%)$ & $33(5.8 \%)$ & $\chi^{2}(2, N=680)=18.11^{* *}$ \\
\hline DAS-prenatal & $M=41.26(S D=6.93)$ & $M=38.66(S D=7.60)$ & $M=41.58(S D=5.48)$ & $F(2,661)=6.38^{* *}$ \\
\hline DAS — birth & $M=42.63(S D=5.09)$ & $M=41.06(S D=5.98)$ & $M=42.64(S D=5.05)$ & $F(2,655)=2.36^{+}$ \\
\hline DAS -6 months & $M=39.74(S D=6.29)$ & $M=38.26(S D=7.63)$ & $M=40.79(S D=5.72)$ & $F(2,645)=4.87^{* *}$ \\
\hline DAS-5 years & $M=40.61(S D=5.36)$ & $M=40.11(S D=5.92)$ & $M=40.48(S D=5.74)$ & $F(2,608)=0.12$ \\
\hline Poverty (low income) & $4(6.6 \%)$ & $9(14.8 \%)$ & $44(7.7 \%)$ & $\chi^{2}(2, N=689)=3.79$ \\
\hline Chronic child illness & $6(9.8 \%)$ & $3(4.9 \%)$ & $55(9.7 \%)$ & $\chi^{2}(2, N=691)=1.50$ \\
\hline Child hospitalizations & $4(6.6 \%)$ & $9(14.8 \%)$ & $64(11.2 \%)$ & $\chi^{2}(2, N=691)=2.11$ \\
\hline Maternal deviance & $5(8.2 \%)$ & $2(3.2 \%)$ & $16(2.8 \%)$ & $\chi^{2}(2, N=669)=4.60^{+}$ \\
\hline Partner deviance & $11(18.0 \%)$ & $21(34.4 \%)$ & $118(20.7 \%)$ & $\chi^{2}(2, N=668)=5.85^{*}$ \\
\hline Maternal prenatal stress & $M=1.79(S D=1.42)$ & $M=2.03(S D=1.57)$ & $M=1.46(S D=1.56)$ & $F(2,672)=4.71^{* *}$ \\
\hline Maternal postnatal stress & $M=1.75(S D=1.64)$ & $M=1.75(S D=1.64)$ & $M=1.18(S D=1.37)$ & $F(2,665)=7.03^{* *}$ \\
\hline Number of adversities & $M=0.51(S D=0.68)$ & $M=0.93(S D=1.01)$ & $M=0.56(S D=0.82)$ & $F(2,643)=5.67^{* *}$ \\
\hline Maternal depressive disorder to age 5 & $16(26.2 \%)$ & $13(21.3 \%)$ & $66(11.6 \%)$ & $\chi^{2}(2, N=688)=12.99^{* *}$ \\
\hline Maternal anxiety disorder to age 5 & $3(4.9 \%)$ & $4(6.6 \%)$ & $18(3.2 \%)$ & $\chi^{2}(2, N=690)=2.13$ \\
\hline
\end{tabular}

${ }^{+} p<.10{ }^{*} p<.05 .{ }^{* *} p<.01$.

children whose mothers reported experiencing more stressors while pregnant were more likely to develop an anxiety disorder $(\mathrm{OR}=1.22,95 \% \mathrm{CI}=1.04-1.43)$. Similarly, maternal postnatal stress also significantly predicted anxiety disorders by adolescence, $\chi^{2}(1, N=607)=5.16$, $p=.02 \mathrm{OR}=1.23,95 \% \mathrm{CI}=1.04-1.46$.

Chronic poverty during the first 5 years was a marginal predictor of an anxiety disorder diagnosis by age 15 , although it was not significant, $\chi^{2}(1, N=625)=$ $3.37, p=.07$. The odds ratio indicated that children living in consistent poverty were approximately twice as likely to meet criteria for an anxiety disorder $(\mathrm{OR}=2.18,95 \%$ $\mathrm{CI}=1.00-4.77)$.
Overall, the total number of adversities experienced in early life significantly predicted later anxiety disorders, $\chi^{2}(1, N=582)=7.67, p<.01$, such that children experiencing a greater number of adverse conditions were more likely to develop anxiety disorders $(\mathrm{OR}=1.50,95 \%$ $\mathrm{CI}=1.14-1.97)$.

\section{Influence of Concurrent Stressors}

We considered the possibility that early adversities might spuriously be associated with later outcomes due to the hidden effects of current (age 15) adverse (stressful)

Table III. Early Adverse Conditions as Predictors of Youth Disorders

\begin{tabular}{|c|c|c|c|}
\hline Variable & $\begin{array}{l}\text { Depressive vs. } \\
\text { anxiety disorder } \\
\text { OR }(\mathrm{CI})\end{array}$ & $\begin{array}{l}\text { Depressive disorder } \\
\text { vs. controls } \\
\text { OR }(\mathrm{CI})\end{array}$ & $\begin{array}{l}\text { Anxiety disorder } \\
\text { vs. controls } \\
\text { OR (CI) }\end{array}$ \\
\hline Partner separation & $1.96(0.80-4.80)$ & $0.94(0.46-1.96)$ & $1.87(1.00-3.51)^{*}$ \\
\hline Change partner & $7.46(1.56-35.72)^{* *}$ & $0.42(0.10-1.87)$ & $3.67(1.74-7.72)^{* *}$ \\
\hline DAS-prenatal & $0.95(0.90-1.00)^{*}$ & $1.00(0.95-1.05)$ & $0.94(0.90-0.98)^{* *}$ \\
\hline DAS—birth & $0.94(0.87-1.02)$ & $1.01(0.95-1.07)$ & $0.96(0.91-1.00)^{+}$ \\
\hline DAS-6 months & $0.97(0.91-1.03)$ & $0.97(0.93-1.02)$ & $0.94(0.90-0.98)^{* *}$ \\
\hline DAS -5 years & $0.98(0.91-1.05)$ & $1.01(0.96-1.06)$ & $0.99(0.95-1.04)$ \\
\hline Maternal deviance & $0.34(0.06-1.88)$ & $2.43(0.84-7.01)$ & $1.00(0.22-4.55)$ \\
\hline Partner deviance & $2.46(1.05-5.77)^{*}$ & $0.75(0.37-1.51)$ & $1.81(1.02-3.22)^{*}$ \\
\hline Poverty & $2.46(0.70-8.62)$ & $0.98(0.33-2.91)$ & $2.18(1.00-4.77)^{*}$ \\
\hline Child hospitalizations & $2.75(0.76-9.98)$ & $0.48(0.16-1.42)$ & $1.26(0.58-2.73)$ \\
\hline Chronic child illness & $0.56(0.13-2.44)$ & $0.94(0.38-2.32)$ & $0.49(0.15-1.62)$ \\
\hline Maternal stress-prenatal & $1.13(0.89-1.44)$ & $1.08(0.92-1.28)$ & $1.22(1.04-1.43)^{*}$ \\
\hline Maternal stress-postnatal & $0.95(0.75-1.21)$ & $1.21(1.01-1.44)^{*}$ & $1.23(1.03-1.46)^{*}$ \\
\hline Number of adversities & $2.00(1.20-3.34)^{* *}$ & $0.85(0.59-1.22)$ & $1.50(1.14-1.97)^{* *}$ \\
\hline
\end{tabular}

${ }^{+} p<.10 .{ }^{*} p<.05 .{ }^{* *} p<.01$. 
conditions. To explore this possibility, three sets of analyses were undertaken. First, we explored the extent to which early adversities might in fact be relatively chronic and continuing. Data on chronic environmental stressors in several domains in the past 6 months were available from interviews of the mother. Correlations between early adversity variables and their current closest counterparts were conducted. Current maternal romantic relationship chronic stress showed small but significant associations with early reports of marital difficulties, including partner separations $(r=.10, p<.01)$, partner changes $(r=.09$, $p<.01)$, and marital satisfaction at the prenatal $(r=$ $-.19, p<.01)$, postnatal $(r=-.24, p<.01), 6$ month $(r=-.26, p<.01)$, and 5 year $(r=-.29, p<.01)$ assessments (where low marital satisfaction was associated with higher marital dissatisfaction). Similarly, maternal report of current level of chronic financial stress was significantly associated with early reports of chronic poverty $(r=.20, p<.01)$. Maternal report of family (including youth) chronic health problems was correlated both with youth early hospitalizations $(r=.07, p=.05)$ and early chronic illness $(r=.09, \quad p=.01)$. Other early adversity variables (e.g., partner crime) did not have current counterparts. Overall, the analyses indicated modest stability of adverse conditions over a 15-year period.

Second, we compared the anxious and depressed groups of youth on current chronic stressful family conditions to test the possibility that early adversity "effects" are really due to group differences in exposure to current adverse conditions that might affect youth diagnoses. The anxiety, depressed, and control youth groups were compared on several current context variables based on the maternal chronic stress interview: maternal marital relationship quality, quality of the mother's relationship with the youth, and family financial stress. There were no significant differences between groups on these variables. Similarly, the groups did not differ in mothers' current marital status (single, married to biological father, married to nonbiological father), $\chi^{2}(4, N=673)=5.35, p>$ .25. Overall, therefore, youth chronic current adversities did not distinguish among the diagnostic groups.

Third, we evaluated the predictive power of early adversities controlling for their contemporary counterpart to see if early adversities retain their unique statistical associations with youth outcomes when the potential effects of current conditions are controlled. Logistic regression analyses were conducted to predict diagnostic outcomes from those early adversity variables for which there was a comparable stress rating available at age 15 . For each regression, current chronic strain was entered with gender and history of maternal depressive and anxiety disor- ders. The patterns of results for differentiating depression and anxiety were the same as in the initial analyses. Both partner changes, $\chi^{2}(1, N=121)=8.78, p<.01$, and prenatal marital dissatisfaction, $\chi^{2}(1, N=117)=4.44$, $p=.04$, were predictive of anxiety disorders over depressive disorders controlling for current maternal marital/relationship stress. Comparing the depressed group to the control group, no early adversities were predictive of developing a depressive disorder even after current stressors were controlled. The pattern of results from comparing the anxiety and control groups was almost identical to results obtained in the previously reported logistic regression analyses. However, although chronic early poverty previously distinguished between anxious and control adolescents, once current financial stress was controlled, early poverty only demonstrated a trend toward predicting anxiety disorder compared to controls, $\chi^{2}(1, N=623)=2.65, p=.10$. Partner changes $\left(\chi^{2}[1\right.$, $N=614]=9.91, p<.01)$ and maternal marital relationship quality prenatally $\left(\chi^{2}[1, N=597]=9.69, p<\right.$ $.01)$ and at 6 months $\left(\chi^{2}[1, N=585]=7.18, p<.01\right)$ significantly differentiated the anxiety group from the control group, controlling for current maternal relationship stress.

\section{DISCUSSION}

The present study was prompted by insufficient information on specificity of the effects of early adverse environmental conditions on depressive outcomes. Many studies, typically retrospective reports, have shown an association between marital discord, stress, and parental psychopathology and depression, but such difficulties have also been linked with a wide array of disorders in youth and adults (e.g., Kessler et al., 1997). Since depressive disorders are likely to co-occur with other disorders, further theoretical advances depend on identification of early adversities that might be specifically related to depression, or another disorder, when the effects of comorbidity are controlled or eliminated. In the present longitudinal study, contemporaneous reports of negative circumstances across five domains were obtained (mother's relationship with partner, economic hardship, early childhood health problems, parental deviance, and maternal stressful life events). Predictive associations between early adversities and "pure" depressive and anxiety disorders in adolescence indicated that youth who developed an anxiety disorder by age 15 were more likely than depressed youth to be exposed to the early negative conditions assessed in this study when each group was compared to controls. This was the case even when analyses controlled for gender and for the effects of maternal depressive and anxiety disorders 
during the early years, which are themselves associated with higher rates of adverse conditions.

Comparisons between the anxiety disorder and depressive disorder groups indicated that the youth with an anxiety disorder were significantly more likely to have been exposed to mothers' partner changes, prenatal marital dissatisfaction, and mothers' partners' trouble with the law. Summing across all adversities, anxiety disorder adolescents were more likely to have experienced a greater number of adversities than were youth who developed depressive disorders. When each youth disorder group was compared with the never ill controls, the depressed youth differed only on more exposure to maternal postnatal stress. In contrast, anxiety disorder youth were significantly more likely than controls to have experienced a wide variety of adverse conditions including marital disorder variables and maternal stressors. Contrary to expectation, there were no associations between childhood chronic illness and hospitalization and anxiety disorder outcomes. However, this may have been a result of the comparison groups employed in the study. Previous research has found inconsistent results for the emotional functioning of children with chronic illness depending on the comparison group selected (e.g., Gartstein, Short, Vannatta, \& Noll, 1999).

Overall, in view of the commonly reported associations among depression and early adversities (Brown \& Harris, 1993; Oakley-Browne, Joyce, Wells, Bushness, \& Hornblow, 1995; Sadowski, Ugarte, Kolvin, Kaplan, \& Barnes, 1999), the present results are somewhat surprising. The current study suggests that if anything, early experiences were more likely to predict anxiety disorders than depression. The present results are consistent with the possibility that prior reports of a link between adversity and depressive outcomes may have been attributable to comorbid anxiety-depression conditions, and to failure to adequately control for maternal depression which is itself highly linked to relationship discord and stressful life events.

The explanation for the closer association of early adversity and anxiety disorders cannot be determined from the present study. Limited retrospective research has suggested that maternal prenatal stress is associated with various child problems including attention deficits, behavioral disorders, and depression, all of which may reflect an underlying dysregulation of the hypothalamic-pituitaryadrenal axis affecting emotionality and responses to stressors (see Mulder et al., 2002 for a review). More specific biopsychological models of the development of anxiety disorders would be needed to address the issue and to distinguish such mechanisms from those hypothesized to eventuate in depressive disorders.
There might also be clinical and developmental explanations for the present results. Depressive disorders generally tend to be more episodic, compared to relatively more stable anxiety disorders. It may be that the latter also have earlier onset in the lives of adolescents. Hence, exposure to chronic adversity in early development may portend early onset and chronic anxiety disorders, whereas depressive disorders are more likely to be triggered by severe acute stressors. Such speculations would be consistent with data on adult women reported by Brown, Harris, and Eales (1993), who found that anxiety disorders were associated only with childhood adversity (but not adult adversity), whereas depressive disorders were associated with both early adversity and contemporary adult stressors, such as losses and poor social support. Cognitive appraisals of early adversities may mediate the link between exposure and later symptoms. It is possible to speculate that the childhood adversities included in the present analyses may have been experienced by the later-anxious child as threatening, leading to generalized cognitions of threat and danger, whereas others who had such experiences did not interpret them as threats. Unfortunately, no information on cognitions was available, and future research exploring the cognitive and biological mechanisms would be fruitful.

Elsewhere we have argued that there are different pathways to adolescent depression depending on maternal depression status (Hammen \& Brennan, 2001). Some youth of depressed mothers, because of their negative family environments, may be vulnerable to the development of depressive disorders given sufficient triggering by personally relevant stressors, whereas other youth might become depressed mainly because of severe levels of stress independent of family conditions. In both instances, the "causes" of their depression may be more proximal to their recent situations, rather than linked to distal experiences of childhood adversity.

Alternately, the lack of prediction of depressive disorders may be attributable to the adversities examined in the current study. Possibly unexamined adverse experiences, such as harsh parenting or neglectful parenting may be more related to depressive than anxiety disorders through their impact on development of appropriate regulation of affect or development of feelings of self-worth or self-competence.

Anxiety disorders are often themselves precursors or risk factors for the development of depressive episodes (Kovacs, Gatsonis, Paulauskas, \& Richards, 1989). It is possible that the current results capture a developmental process in which early adversity portends early onset anxiety disorders - which themselves might predict eventual depressive disorders. Thus, for many individuals, early 
adverse experiences may eventuate in depressive disorders through their initial effects predicting anxiety disorders. Since the youth of the present study are only 15 years of age, they have ample opportunity to develop depressive disorders as they age. A further follow-up of the youth which is underway may help to address the question of whether "pure" anxiety disorders in those exposed to childhood adversity may increase the likelihood of depressive reactions to stress over time.

Several limitations of this study should be considered in evaluating these results. First, the data on early adversities were limited by their reliance on maternal selfreport data. Such information had the advantage that it was current at the time, but it has the disadvantage that no independent ascertainment of the validity of reports was available. During the original study only a relatively small number of questions were asked about each domain of interest, and little information was available to quantify the severity of a woman's experience. Additionally, several potential early adversities of interest, such as sexual and physical abuse, parental death and family violence, were either not collected or were too infrequent in the sample to be included. The range of available adversities may have excluded content that would be relevant to differential prediction of depression and anxiety disorders. Finally, the adversities assessed were limited to those occurring by child's age 5 , and it is possible that negative environmental experiences occurring later in childhood are also highly relevant to onset of depression or anxiety disorders.

Similarly, inaccuracies in recall of symptoms and disorders may have occurred. As Macia et al. (2003) indicated, some individuals may not recall having had a disorder when they actually did. However, reliance on both youth and mother interviews may have reduced the likelihood that both would have overlooked disorders. A further limitation concerns the relatively small samples in the individual diagnostic groups, especially the pure anxiety group, which may have limited ability to detect reliable variations in early adversities. Also, it is acknowledged that relatively pure depressive or anxiety disorders are not typical due to high levels of comorbidity. While generalizability to clinical samples may thereby be limited, the conceptual issues addressed by the study required such a strategy.

Results from this exploratory study of differences between depressive and anxiety disorders in adolescence indicate that anxiety disorders are more strongly associated with early adversities to age 5 than is depression, even when controlling for maternal anxiety and depression status. Although the inability to predict depressive disorders from early adversity is unexpected given previously published findings, these findings present an interesting picture of the potential differences in the development of anxiety and depressive disorders. More research is needed to explore the mechanisms by which early adversities influence later outcomes of specific psychiatric disorders directly, and to evaluate their development further as the adolescents experience the transition to adulthood.

\section{ACKNOWLEDGMENTS}

This study was supported by funds from the National Health and Medical Research Council in Australia, the Mater Misericordiae Mother's Hospital in Queensland, Australia and NIH Grant R01 MH52239. We are grateful to the Brisbane project coordinators of the age 15 follow-up, Robyne LeBrocque and Cheri Dalton, and interviewers Barbara Mann, Eileen Tone, Sandra Fergusson, Molly Robbins, and Lisa Manning. We also thank Margaret Andersen, Michael O'Callaghan, and Gail Williams for their contributions to the original study of the MUSP birth cohort.

\section{REFERENCES}

Allen, N. B., Lewinsohn, P. M., \& Seeley, J. R. (1998). Prenatal and perinatal influences on risk for psychopathology in childhood and adolescence. Development and Psychopathology, 10, 513-529.

Bandelow, B., Spaeth, C., Alvarez Tichauer, G., Broocks, A. Hajak, G., \& Ruether, E. (2002). Early traumatic life events, parental attitudes, family history, and birth risk factors in patients with panic disorder. Comprehensive Psychiatry, 43, 269-278.

Beck, A., Brown, G., Steer, R., Eidelson, J., \& Riskind, J. (1987). Differentiating anxiety and depression: A test of the cognitive contentspecificity hypothesis. Journal of Abnormal Psychology, 96, 179183.

Bedford, A., Foulds, G. A., \& Sheffield, B. F. (1976). A new personal disturbance scale (DSSI/SAD). British Journal of Social and Clinical Psychology, 15, 387-394.

Brown, G. W., \& Harris, T. O. (1993). Aetiology of anxiety and depressive disorders in an inner-city population. 1. Early adversity. Psychological Medicine, 23, 143-154.

Brown, G. W., \& Harris, T. O., \& Eales, M. J. (1993). Aetiology of anxiety and depressive disorders in an inner-city population. 2 . Comorbidity and adversity. Psychological Medicine, 23, 155-165.

Burbach, D., \& Borduin, C. (1986). Parent-child relations and the etiology of depression: A review of methods and findings. Clinical Psychology Review, 6, 133-153.

Coie, J., Watt, N., West, S., Hawkins, J., Asarnow, J., Markman, H., et al. (1993). The science of prevention: A conceptual framework and some directions for a national research program. American Psychologist, 48, 1013-1022.

Cohen, P., Velez, C. N., Brook, J., \& Smith, J. (1989). Mechanisms of the relation between perinatal problems, early childhood illness, and psychopathology in late childhood and adolescence. Child Development, 60, 701-709.

Finlay-Jones, R., \& Brown, G. (1981). Types of stressful life event and the onset of anxiety and depressive disorders. Psychological Medicine, $11,803-815$.

First, M. B., Spitzer, R. L., Gibbon, M., \& Williams, J. B. W. (1995). Structured clinical interview for DSM-IV axis I disorders. Washington: American Psychiatric Press. 
Fredman, S., Hirshfeld-Becker, D., Smoller, J., \& Rosenbaum, J. (2003). Childhood antecedents of adult anxiety disorders. In D. Nutt \& J. Ballenger (Eds.), Anxiety disorders (pp. 135-161). Malden, MA: Blackwell Science.

Gartstein, M. A., Short, A. D., Vannatta, K., \& Noll, R. B. (1999). Psychosocial adjustment of children with chronic illness: An evaluation of three models. Journal of Developmental \& Behavioral Pediatrics, $20,157-163$.

Goodman, S. (2002). Depression and early adverse experiences. In I. Gotlib \& C. Hammen (Eds.), Handbook of depression (pp. 245267). New York: Guilford Press.

Gortmaker, S. L., Walker, D. K., Weitzman, M., \& Sobol, A. M. (1990). Chronic conditions, socioeconomic risks, and behavioral problems in children and adolescents. Pediatrics, 85, 267-276.

Hammen, C. (1991). Depression runs in families: The social context of risk and resilience in children of depressed mothers. New York, NY: Springer-Verlag.

Hammen, C., \& Brennan, P. (2001). Depressed adolescents of depressed and nondepressed mothers: Tests of an interpersonal impairment hypothesis. Journal of Consulting and Clinical Psychology, 69, 284-294.

Hammen, C., Adrian, C., Gordon, D., Burge, D., Jaenicke, C., \& Hiroto, D. (1987). Children of depressed mothers: Maternal strain and symptom predictors of dysfunction. Journal of Abnormal Psychology, 96, 190-198.

Johnson, J. G., Cohen, P., Kasen, S., Smailes, E., \& Brook, J. S. (2001). Association of maladaptive parental behavior with psychiatric disorder among parents and their offspring. Archives of General Psychiatry, 58, 453-460.

Kaslow, N. J., Deering, C. G., \& Racusin, G. R. (1994). Depressed children and their families. Clinical Psychology Review, 14, 39-59.

Keeping, J. D., Najman, J. M., Morrison, J., Western, J. S., Andersen, M. J., \& Williams, G. M. (1989). A prospective longitudinal study of social, psychological, and obstetrical factors in pregnancy: Response rates and demographic characteristics of the 8,556 respondents. British Journal of Obstetrics and Gynaecology, 96, 289-297.

Kellerman, J., Zeltzer, L., Ellenberg, L., Dash, J., \& Rigler, D. (1980). Psychological effects of illness in adolescence: 1 . Anxiety self-esteem and perception of control. Journal of Pediatrics, 97,126-131.

Kessler, R. C., \& Magee, W. J. (1993). Childhood adversities and adult depression: Basic patterns of association in a US national survey. Psychological Medicine, 23, 679-690.

Kessler, R. C., Davis, C. G., \& Kendler, K. S. (1997). Childhood adversity and adult psychiatric disorder in the US National Comorbidity Survey. Psychological Medicine, 27, 1101-1119.

Kovacs, M., Gatsonis, C., Paulauskas, S. L., \& Richards, C. (1989). Depressive disorders in childhood: IV. A longitudinal study of comorbidity with and risk for anxiety disorders. Archives of General Psychiatry, 46, 776-782.

Laraia, M. T., Stuart, G. W., Frye, L. H., Lydiard, R. B., \& Ballenger, J. C. (1994). Childhood environment of women having panic disorder with agoraphobia. Journal of Anxiety Disorders, 8, 1-17.
Leckman, J., Sholomskas, D., Thompson, W., Belanger, A., \& Weissman, M. (1982). Best estimate of lifetime psychiatric diagnoses: A methodological study. Archives of General Psychiatry, 39, 879-883.

Levitan, R., Rector, N., Sheldon, T., \& Goering, P. (2003). Childhood adversities associated with major depression and/or anxiety disorders in a community sample of Ontario: Issues of comorbidity and specificity. Depression and Anxiety, 17, 34-42.

Macia, C., Storch, E., Dent, H., Adams, P., Verdeli, H., Davies, M., \& Weissman, M. (2003). Recall of childhood psychopathology more than 10 years later. Journal of the Academy of Child and Adolescent Psychiatry, 42, 6-12.

Mulder, E. J. H., Robles de Medina, P. G., Huizink, A. C., Van den Bergh, B. R. H., Buitelaar, J. K., \& Visser, G. H. A. (2002). Prenatal maternal stress: Effects on pregnancy and the (unborn) child. Early Human Development, 70, 314.

Oakley-Browne, M. A., Joyce, P. R., Wells, J. E., Bushnell, J. A., \& Hornblow, A. R. (1995). Adverse parenting and other childhood experience as risk factors for depression in women aged 18-44 years. Journal of Affective Disorders, 34, 13-23.

Orvaschel, H. (1995). Schedule for Affective Disorders and Schizophrenia for School-Age Children Epidemiologic Version-5. Ft. Lauderdale, FL: Center for Psychological Studies, Nova Southeastern University.

Rao, U., Hammen, C., \& Daley, S. (1999). Continuity of depression during the transition to adulthood: A 5-year longitudinal study of young women. Journal of the American Academy of Child and Adolescent Psychiatry, 38, 908-915.

Rapee, R. M. (1997). Potential role of childrearing practices in the development of anxiety and depression. Clinical Psychology Review, 17, 47-67.

Repetti, R. L., Taylor, S. E., \& Seeman, T. E. (2002). Risky families: Family social environments and the mental and physical health of offspring. Psychological Bulletin, 128, 230-366.

Sadowski, H., Ugarte, B., Kolvin, I., Kaplan, C., \& Barnes, J. (1999). Early life family disadvantages and major depression in adulthood. British Journal of Psychiatry, 174, 112-120.

Spanier, G. B. (1976). Measuring dyadic adjustment: New scales for assessing the quality of marriage and similar dyads. Journal of Marriage and the Family, 38, 15-28.

Spence, S., Najman, J., Bor, W., O'Callaghan, M., \& Williams, G. (2002). Maternal anxiety and depression, poverty and marital relationship factors during early childhood as predictors of anxiety and depressive symptoms in adolescence. Journal of Chlid Psychology and Psychiatry, 43, 457-469.

Tweed, J. L., Schoenbach, V. J., George, L. K., \& Blazer, D. G. (1989). The effects of childhood parental death and divorce on six-month history of anxiety disorders. British Journal of Psychiatry, 154, 823-828.

Young, E., Abelson, J., Curtis, G., \& Nesse, R. (1997). Childhood adversity and vulnerability to mood and anxiety disorders. Depression and Anxiety, 5, 66-72. 